\title{
Biotope et description de Niphargus altagahizi n. sp., Amphipode Gammaridé souterrain du LIBAN
}

\author{
par
}

N. ALOUF*

A une vingtaine de kilomètres au Sud de Dahr Al'ayn et dans la même couche géologique, se trouve une petite grotte située presque au bord de la Méditerranée (fig. 1); elle a reçu le nom de Ras Chekka (cap de Shikka ou de Sikkâ) par H. Coiffait; les villageeois l'appellent Magharit-L-Mascat (grotte du Précipice). Des prospections effectuées plus récemment ont permis la découverte, dans cette cavité, d'un Amphipode Gammaridé, anophtalme, appartenant au genre Niphargus, qui vient, dans la faune du Liban, s'ajouter à Niphargus nadarini Alouf 1972, découvert dans le village de Hawsh Hala.

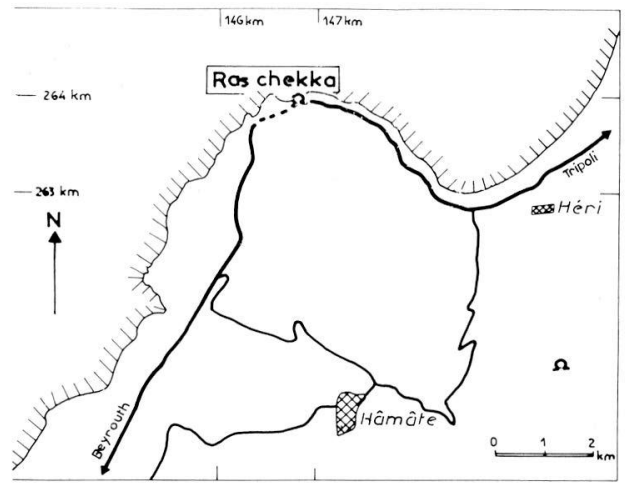

Fig. 1. Situation géographique de la grotte de Ras Chekka.

\section{DONNÉES ÉCOLCIGIQUES SUR LA GROTTE DE RÂS CHEKKA}

Coordonnées : $\mathrm{X}=14660, \mathrm{Y}=26395$ de la carte topographique au $1 / 20000$ ème (feuille de Batroun). Altitude: quelques mètres à l'entrée, zéro mètre au point le plus bas.

Distance du rivage: environ 50 mètres.

Longueur: environ 40 mètres.

Orientation: E.-W., suivant la cassure du massif.

* Faculté des Sciences, Université libanaise, HADETH-BEYROUTH (Liban).

Biologie Souterraine Université Claude Bernard (LYON-I). 
Entrée: assez grande et protégée du soleil par le massif rocheux qui avance en pointe à l'Ouest. Ainsi, l'entrée de la grotte est protégée des rayons solaires en hiver; mais, en été, ces rayons pénètrent profondément dans la grotte lors du coucher du soleil (voir fig. 2).

Intérieur: La grotte a un petit lac inférieur, à zéro mètre d'altitude (fig. 3). L'influence de la mer s'y fait sentir; ainsi le niveau et la superficie de ce petit lac varient suivant le cycle des marées.

Température : Air $=$ de $13^{\circ} 2$ en hiver, à $20^{\circ} 3$ en été.

$E a u=$ la température subit une oscillation annuelle particulière à chaque endroit de la grotte; ces oscillations influenceraient la répartition de la faune. Ainsi la température oscille entre $17^{\circ} 1$ et $19^{\circ}$ dans le gours le plus haut G1, et entre $14^{\circ} 3$ et $19^{\circ}$ dans le lac inférieur (voir tableau 1).

Salinité: l'eau est douce, bien que l'influence de la mer se fasse sentir dans le lac inférieur: l'eau douce ne ferait que surmonter sans mélange l'eau salée plus dense. L'analyse de la conductivité de l'eau a donné les chiffres suivants:

\begin{tabular}{l|l|l|l|l|l}
\hline Date & G1 & G4 & G5 & G5 & mer \\
\hline $7-3-72$ & $\begin{array}{l}0,84 \text { Mho } \\
\text { 0,94 Mho }\end{array}$ & 0,84 & 0,90 & $\begin{array}{l}0,91(-40 \mathrm{~cm}) \\
1,14(-60 \mathrm{~cm})\end{array}$ & 60
\end{tabular}

Tableau 1.

Variations de la température dans la grotte.

\begin{tabular}{l|l|l|l|l|l|l|l}
\hline \multicolumn{1}{r|}{ Stations } & G1 & G2 & G3 & G4 & G5 & Air & (mer) \\
\hline Date & & & & & & & \\
\hline $7-1-7211 \mathrm{~h}$. & 17,4 & 16,5 & 16 & 16 & 15 & 13,2 & 16,3 \\
$10-2-7215 \mathrm{~h}$. & 17,1 & 16,3 & 16 & & 14,3 & 13,3 & 16 \\
$7-3-7212 \mathrm{~h}$. & 18 & 17,6 & 17,3 & 17 & 16 & 15,3 & 16,6 \\
& & & & & & & \\
$11-5-7212 \mathrm{~h}$. & 18,5 & 18,3 & 18,2 & & 17,5 & 17,8 & 19,9 \\
$6-6-72$ & 18,8 & 18,8 & 18,7 & 18,6 & 18,3 & 18,7 & 23,9 \\
$7-7-72$ & 18,9 & 18,9 & 18,9 & 18,9 & 18,85 & 20,2 & 26 \\
$4-8-7211 \mathrm{~h}$. & 19 & 19 & 19 & 19 & 19 & 20,3 & 27,8 \\
& & & & & & & \\
$5-10-7211 \mathrm{~h} .30$ & 19 & 19 & 19 & 19,05 & 19,1 & 20 & 26,5 \\
$5-11-7211 \mathrm{~h}$. & 18,6 & 18,5 & 18,4 & 18,35 & 18,3 & 18,5 & 23,5
\end{tabular}




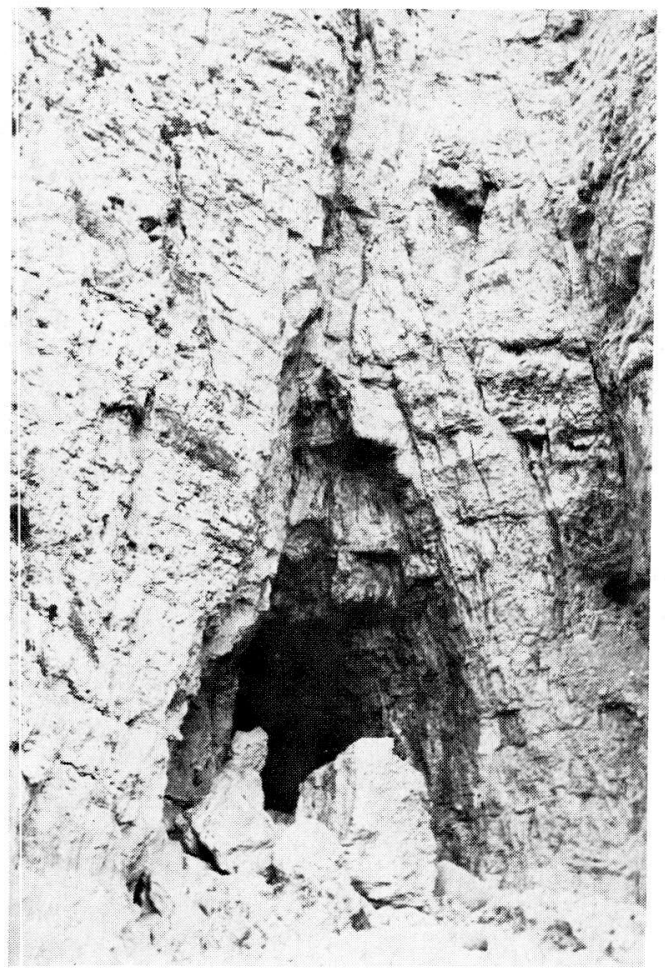

Fig. 2. Entrée de la giotte de Ras Chekka.
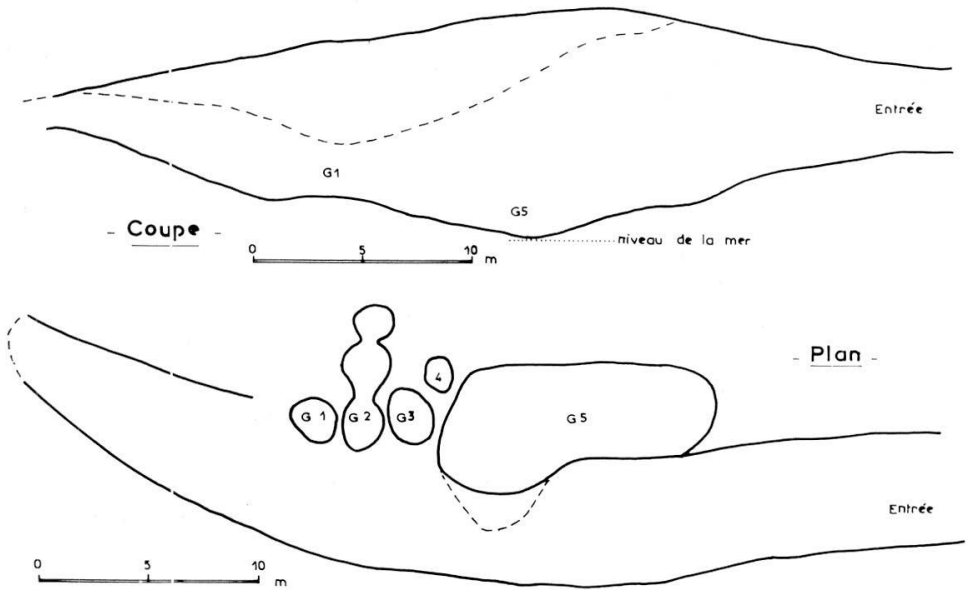

Fig. 3. Plan et coupe schématiques de la grotte de Ras Chekka. 
Il a fallu ajouter 0,56 cc d'eau de mer (prise le 6-6-72) à $250 \mathrm{cc}$ de l'eau prise en G1, pour obtenir la même salinité que celle de l'eau prise en G5 à $60 \mathrm{~cm}$ de profondeur. Les conditions, lors de l'analyse de la conductivité, n'étant pas les mêmes, les chiffres obtenus pour $\mathrm{G} 1$ accusent une différence aux différentes dates.

Géologie: la grotte de Ras Shikka se trouve dans une région où le massif du Liban parvient jusqu'à la mer, sous la forme d'une falaise abrupte de calcaire Miocène. Elle doit être d'origine assez récente, vu l'absence de concrétions de calcite, qui existent pourtant dans d'autres grottes chaudes et humides du littoral libanais.

Cette station est pauvre en Amphipodes. Elle fut prospectée durant l'été 1965. Une pêche au filet à plancton a fourni alors un jeune Amphipode anophtalme et dépigmenté, long de $3 \mathrm{~mm}$, des Cyclopides, des Harpacticides, des Ostracodes, des Hydracariens, des Oligochètes, des Collemboles et un Koenenia.

Le 16-8-66, il fut procédé à une pêche au filet à plancton, et à la mise de balances dans le lac inférieur; ceci n'a rien donné. Mais la balance, mise dans un gour supérieur, a fourni trois Amphipodes anophtalmes, de $6 \mathrm{~mm}, 8 \mathrm{~mm}$ et $9 \mathrm{~mm}$. Les recherches effectuées durant 1970 et 1971 ont fourni 5 autres individus.

\section{DESCRIPTION DE N. ALTAGAHIZI*}

La présente description** est basée sur la dissection de 7 individus tués et conservés dans l'alcool à $70^{\circ}$. Un seul spécimen complet est gardé dans le laboratoire de Sciences Naturelles de l'Université Libanaise (Hadeth-Beyrouth) (fig. 4).

$\mathrm{Vu}$ le nombre peu important d'individus, la taille donnée pour l'espèce sera la taille moyenne des grands individus: $8 \mathrm{~mm}$. Le plus grand spécimen mesure $9 \mathrm{~mm}$. entre la base de l'antenne et la base du telson.

Prosome:

Antenne I. La longueur totale atteint approximativement la moitié de celle du corps. Les flagelles ont entre 21 et 25 articles, la moyenne est de 24 articles. Le flagelle accessoire est biarticulé.

Antenne II. Flagelle formé de 8 articles, mais on trouve des flagelles de 6 et 7 articles.

Maxille I. Le lobe interne porte 1 ou 2 soies terminales et est couvert de fines soies latérales. Le lobe externe a 7 épines, la plus interne a un nombre de dents variant entre 6 et 9. La moyenne est 7; l'épine suivante a un nombre de dents variant entre 2 et 5 , la moyenne est de 4 dents. Les autres épines ont une seule dent. Le dernier article du palpe porte de 4 à 6 soies.

* Al Djahid ou Al Gahiz, érudit arabe du IX siècle, auteur du livre "de l'animal", reçut son nom de Djahiz à cause de ses yeux proéminents. Il a pris une part active au mouvement Mu'tazila qui prêchait la primauté de la science et de la raison sur la religion et la foi. Alta: initiales d'une personne qui m'est chère.

** Je remercie MM. Ginet et Sket qui ont bien voulu lire la présente note et suggérer les corrections et compléments nécessaires. 


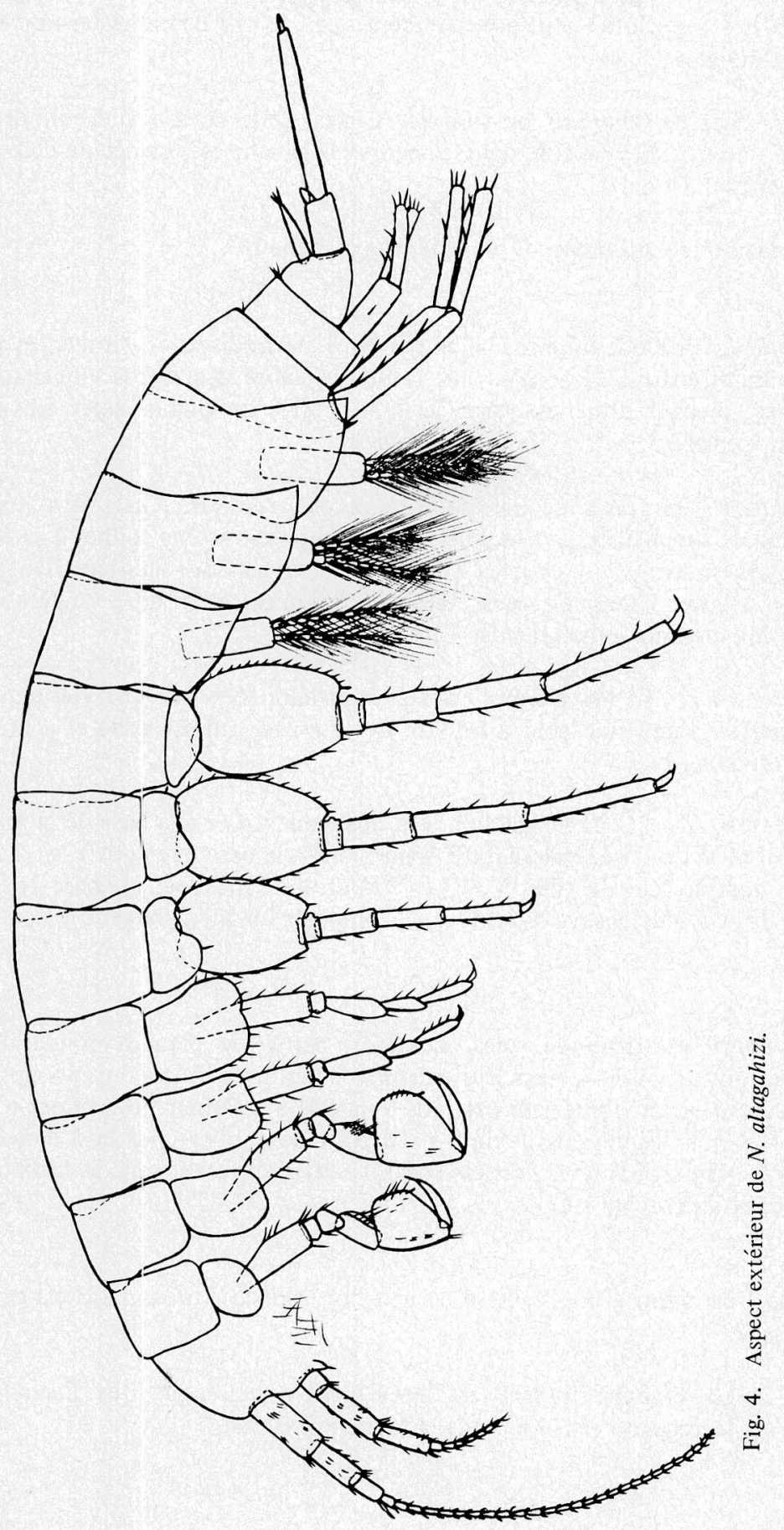


Maxille II. Les deux lobes sont presque identiques, ils ont des soies terminales et de fines soies latérales..

Mandibules. Les pars molaris ne sont pas égales; celle de la mandibule droite est formée de deux parties de cinq dents chacune, la gauche est formée de deux parties de deux dents chacune.

Maxillipède et lèvre inférieure: Voir schémas, planche II.

\section{Mésosome:}

Gnathopode I. Propode trapézoïdal. Sur le bord sternodistal on trouve un nombre d'épines variant entre 3 et 5 , dont une seule est glabre, les autres sont dentées. Le bord sternal, presque droit, est garni de 4 - 4 touffes de longues soies. Les soies du carpos sont pennées.

Gnathopode II. Le propode est plus grand que celui du Gn1, il a un aspect trapézoïdal, le bord distal est incliné. L'épine repose sur une saillie; à sa base, du côté sternal, on trouve 3 courtes épines, dont deux sont dentées. Du côté du dactyle on trouve 3 longues soies. Le bord sternal est garni de 2 rangées de soies courtes et longues, aussi bien dans le Gn1 que Gn2.

Péréiopodes III et IV. Les plaques coxales sont allongées. Les dactyles sont garnis d'une épine et d'une fine soie à la base de la griffe, qui est grêle et a presque la longueur du dactyle.

Péréiopodes V, VI et VII. Le dactyle $\mathrm{V}$ a une seule épine à la base de la griffe. Les dactyles VI et VII ont généralement 2 épines: une à la base de la griffe, et l'autre au milieu du dactyle. Les dactyles V, VI et VII ont une fine soie à la base de la griffe. Le basis du P7 n'est pas très allongé, le rapport longueur/largeur est d'environ $12 / 10$.

\section{Métasome:}

Le bord dorsal est garni de soies. Les trois paires de plaques épimérales sont pointues et ont une soie forte à leur extrémité. Les deux fouets des pléopodes sont de longueurs inégales, l'intérieur est plus long que l'extérieur; le nombre d'articles est de $8-9$ pour l'intérieur et de $9-10$ pour l'extérieur chez la 3 ème paire et sont dans le rapport $8 / 7$ avec l'article basal. Le rétinacle est formé de deux crochets pluridentés et un crochet simple.

\section{Urosome:}

L'urosome I est garni d'une seule soie sur le bord dorsal, l'urosome II est garni de 3 ou 4 soies.

Uropode I. La branche interne est légèrement plus longue que l'article basal; lui-même est légèrement plus long que la branche externe. 

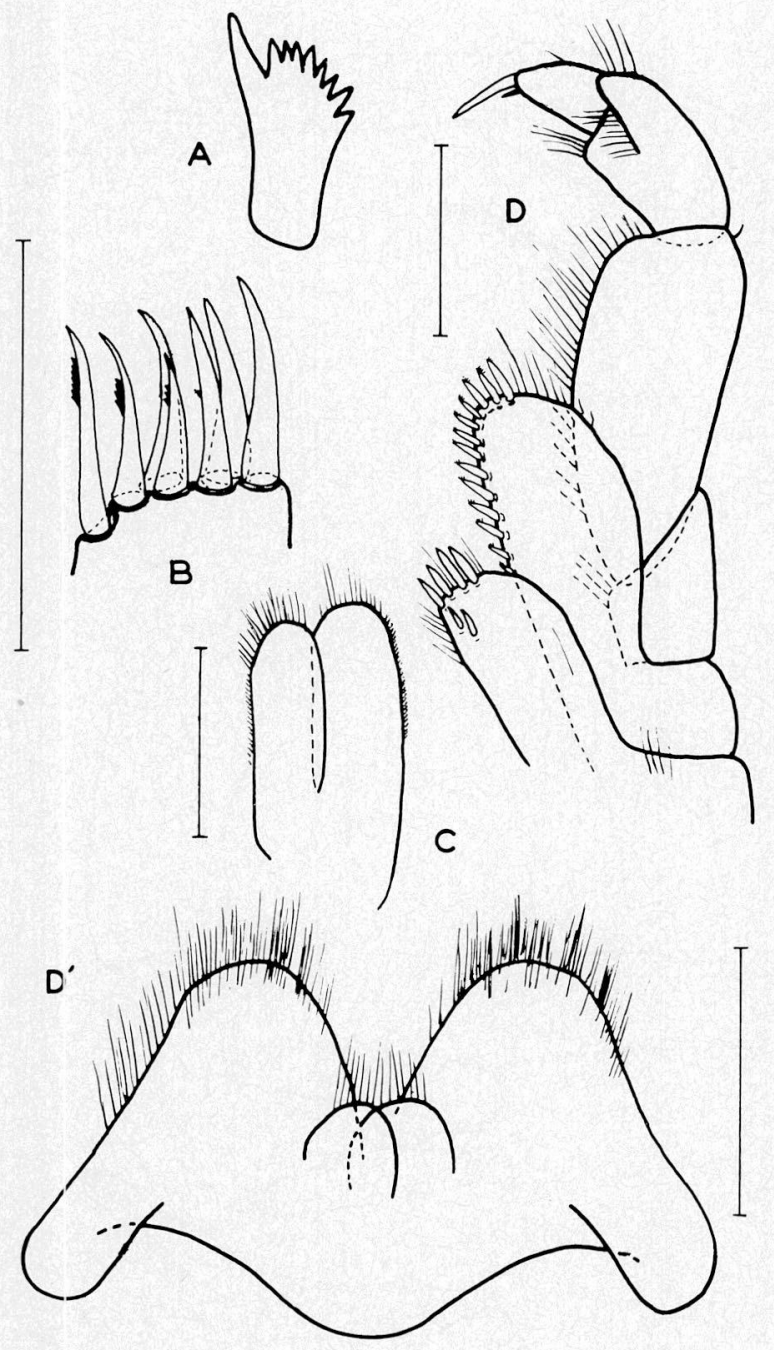

PLANCHE 1.

A. - Lacinia mobilis,

B. - Lobe externe du Maxille I (individu de $8,8 \mathrm{~mm}$.),

C. - Maxille II,

D. - Maxillipède,

D'.- Lèvre inférieure. 


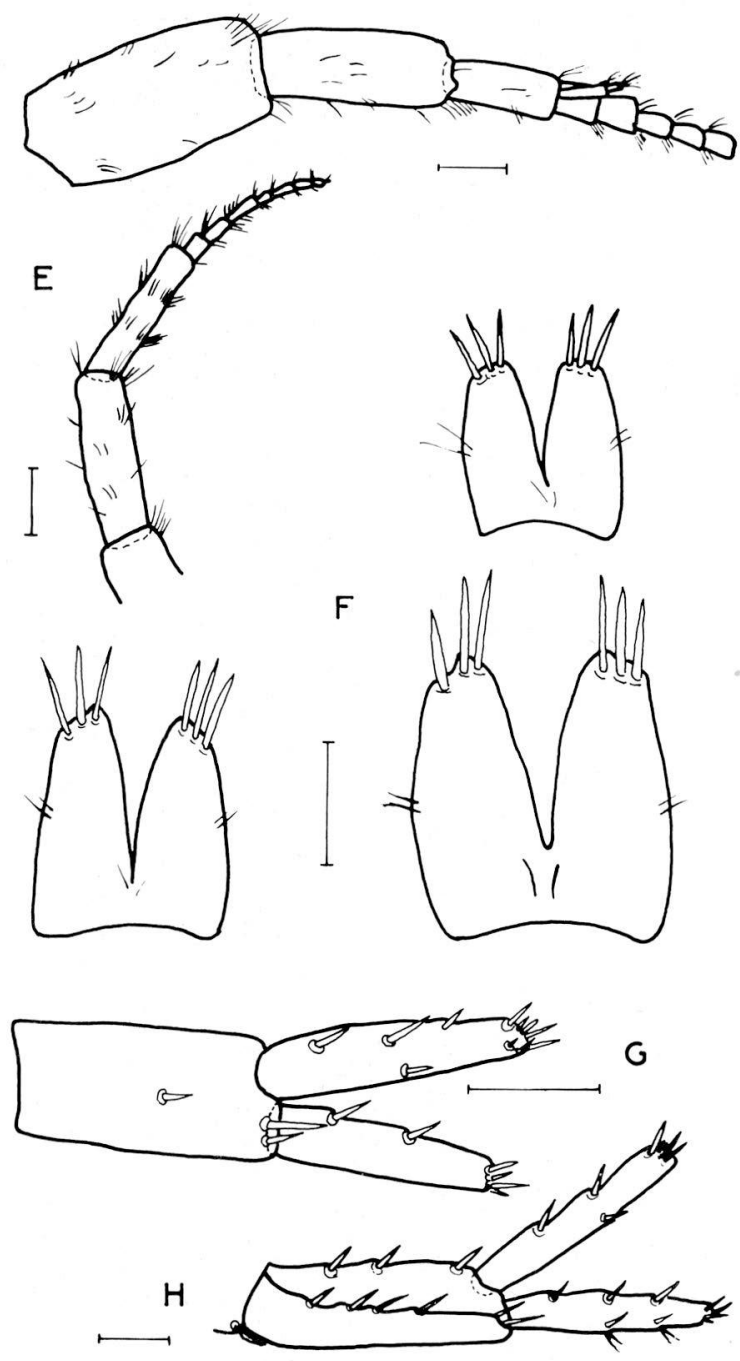

PLANCHE 2.

E. - Antennes I (fragment basal) et II,

F. - Telsons,

G. - Uropode II (ind. de $8 \mathrm{~mm}$.),

H. - Uropode I (ind. de $8 \mathrm{~mm}$.), 


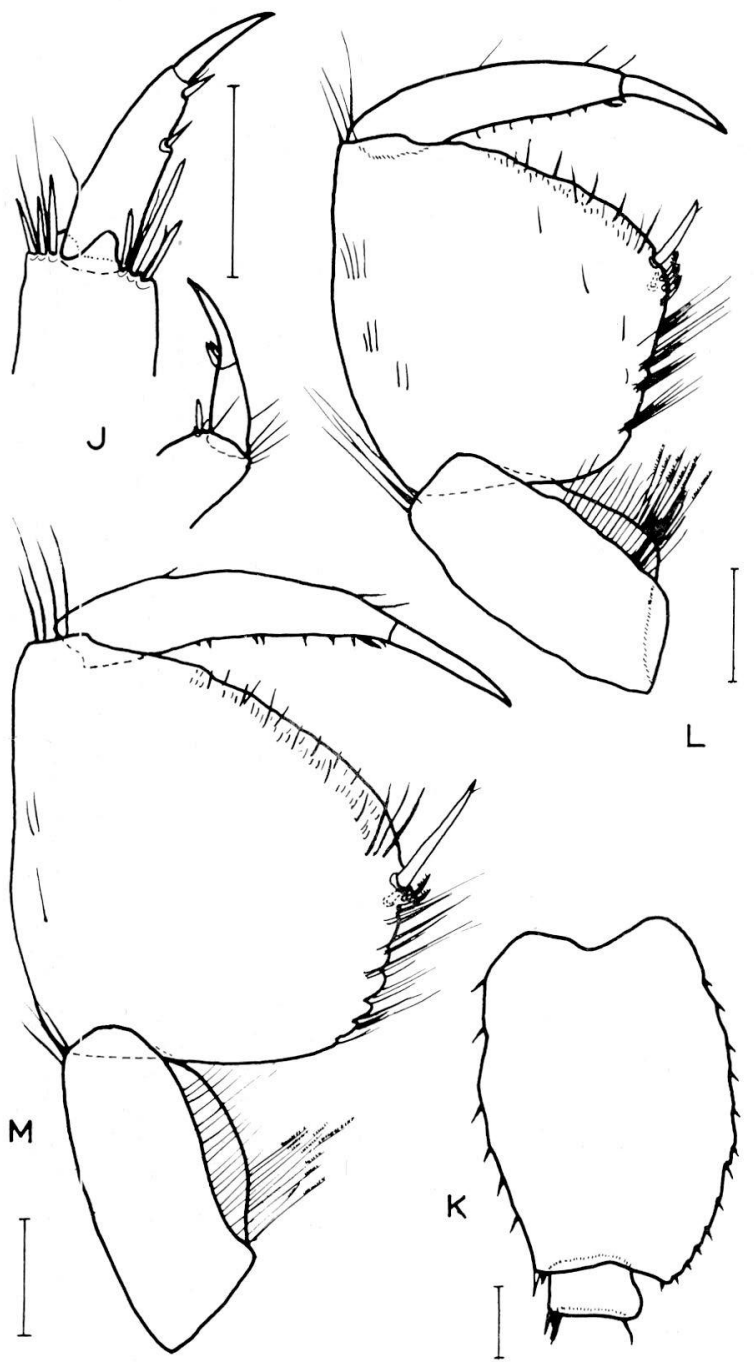

PLANCHE 3.

J. - Dactyle des péréiopodes VII et IV,

K. - Basis (ind. de $8 \mathrm{~mm}$.),

L. - GnI (ind. de $8,8 \mathrm{~mm}$.),

M. - Gn II (ind. de $8,8 \mathrm{~mm}$.). 

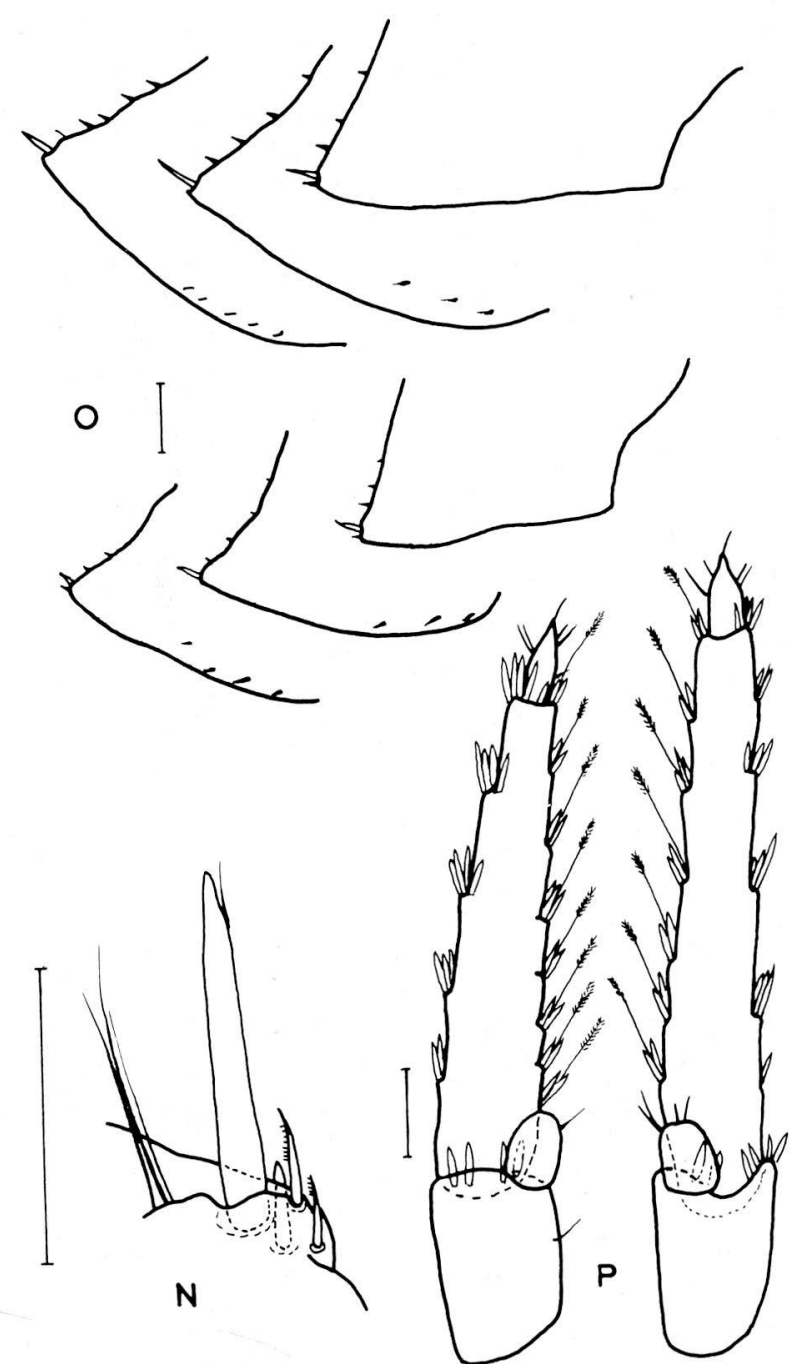

PLANCHE 4.

N. - Epine palmaire (ind. de $8,8 \mathrm{~mm}$.),

O. - Plaques épimérales (ind. de $8,8 \mathrm{~mm}$. et de $8 \mathrm{~mm}$.),

P. - Uropodes III (ind. de $8 \mathrm{~mm}$.).

(Pour toutes les planches, le segment à côté de chaque dessin représente une longueur de $0,2 \mathrm{~mm}$.). 
Uropode II. La branche interne est plus longue que la branche externe. Cette dernière est égale ou plus petite que l'article basal.

Uropode III. L'article distal de la branche externe est petit, il est indifférencié chez le mâle et la femelle.

\section{Telson:}

Plus long que large, la base est bombée et large. Il est échancré jusqu'aux 3/4. Les lobes sont terminés par 3 épines. On rencontre deux soies sur le bord extérieur des lobes et deux finess soies à la base de l'échancrure.

\section{DISCUSSION}

Les deux espèces libanaises, Niphargus nadarini Alouf 1972 et Niphargus altagahizi n. $s p$. sont très différentes l'une de l'autre, bien qu'elles vivent dans des régions distantes seulement de $60 \mathrm{Km}$ à vol d'oiseau. Pour la même longueur du P7, les individus ont une taille assez proche; mais les rapports entre les organes sont différents (tableau 2).

Les deux espèces libanaises appartiendraient au groupe orcinus, caractérisé par des plaques coxales allongées (P III et P IV), des plaques épimérales pointues, des gnathopodes trapézoïdaux, le gnathopode II plus important que le I, l'exopodite de l'uropode II plus court que l'endopodite, et l'article distal de l'uropode III court et indifférencié chez le mâle et la femelle.

Ce groupe colcnise essentiellement la Yougoslavie, et se rencontre en Europe occidentale, en Italie (Ruffo et Vigna-Taglianti, 1968) et en Crimée (Birstein, 1961).

Niphargus altagahizi se rattacherait à Niphargus brevicuspis Schell. et Niphargus zavalanus Kar. Ces deux espèces, bien que ne faisant pas partie du groupe orcinus s.s., forment une lignée qui lui est rattachée. $N$. altagahizi se rattacherait aussi à Niphargus kolombatovici Kar. Toutes ces espèces sont caractérisées par leur taille relativement petite (de l'ordre de $10 \mathrm{~mm}$.) et par le rapport antenne/taille ne dépassant pas la valeur $1 / 2$; la forme du basis du $\mathrm{P} 7$, chez $N$. altagahizi, serait proche de celle de $N$. brevicuspis, de même que la forme des gnathopodes, tandis que le telson est plutôt à rapprocher de celui du $N$. zavalanus.

La découverte de deux nouvelles espèces de Niphargus au Proche-Orient nous incite à revoir les frontières de ce genre. Au cas où il serait une relicte des mers anciennes, on devra normalement le retrouver à l'emplacement de ces mers, comme c'est le cas au Litıan: on pourra ainsi peut-étre, élargir le domaine du Niphargus grâce à de nouvelles recherches.

\section{RÉSUMÉ}

Description d'une petite grotte chaude située presque au bord de la Méditerranée; la température de l'eau varie entre 14 et $19^{\circ}$ C. Elle héberge un Niphargus, $N$. 
Tableau 2.

Différences de quelques indices morphologiques entre $N$. altagahizi et $N$. nadarini.

\begin{tabular}{|c|c|c|}
\hline & Niphargus nadarini & Niphargus altagahizi \\
\hline $\begin{array}{l}\text { Taille du corps } \\
\text { (P7 de même } \\
\text { longueur) }\end{array}$ & $10 \mathrm{~mm}$ & $8,8 \mathrm{~mm}$ \\
\hline $\begin{array}{l}\text { Flagelle de } \\
\text { l'antenne II } \\
\end{array}$ & $9-12$ articles & 6-8 articles \\
\hline $\begin{array}{l}\text { GnI } \\
\text { (Propode) }\end{array}$ & $\begin{array}{l}3 \text { épines, dont } 1 \text { gla- } \\
\text { bre; } 9-11 \text { touffes de } \\
\text { longues soies; bord } \\
\text { sternal légèrement } \\
\text { courbé. }\end{array}$ & $\begin{array}{l}\text { 3-5 épines dont } 1 \text { gla- } \\
\text { bre; } 3-5 \text { touffes de } \\
\text { longues soies; bord } \\
\text { sternal plutôt droit. }\end{array}$ \\
\hline $\begin{array}{l}\text { GnII } \\
\text { (Propode) }\end{array}$ & $\begin{array}{l}\text { forme triangulaire; } \\
2 \text { épines dont } 1 \text { den- } \\
\text { tée; } 10-12 \text { touffes de } \\
\text { longues soies. }\end{array}$ & $\begin{array}{l}\text { forme trapézoïdale; } \\
3 \text { épines dont } 2 \text { den- } \\
\text { tées; } 6-7 \text { touffes de } \\
\text { longues soies. }\end{array}$ \\
\hline $\begin{array}{l}\text { Rapport } \\
\text { GnI/GnII } \\
\end{array}$ & $\begin{array}{l}=2 \text { (ind. de } 10 \mathrm{~mm}) \\
=1,7 \text { (grands individus) }\end{array}$ & $=1,5$ \\
\hline $\begin{array}{l}\text { Péréiopodes } \\
\text { III et IV }\end{array}$ & $\begin{array}{l}\text { Plaques coxales : car- } \\
\text { rées à légèrement rec- } \\
\text { tangulaires; griffe III } \\
\text { plutôt courte et trapue }\end{array}$ & $\begin{array}{l}\text { plaques coxales rec- } \\
\text { tangulaires; griffe } \\
\text { III plutôt longue } \\
\text { et fine. }\end{array}$ \\
\hline $\begin{array}{l}\text { Péréiopode } \\
\text { III }\end{array}$ & $\begin{array}{l}\text { Généralement } 1 \text { épine } \\
\text { sur le dactyle, parfois } \\
2 .\end{array}$ & $\begin{array}{l}2 \text { épines sur le dac- } \\
\text { tyle. }\end{array}$ \\
\hline Rétinacles & $\begin{array}{l}2 \text { crochets pluri- } \\
\text { dentés. }\end{array}$ & $\begin{array}{l}2 \text { crochets pluriden- } \\
\text { tés, plus } 1 \text { crochet } \\
\text { simple. }\end{array}$ \\
\hline Telson & $\begin{array}{l}\text { Echancré aux } 4 / 5 ; \\
\text { généralement } 4 \text { épines } \\
\text { terminales, parfois } 5 \text {, } \\
\text { rarement } 3 \text { ou } 6 .\end{array}$ & $\begin{array}{l}\text { échancré aux } 2 / 3 \\
3 \text { épines terminales. }\end{array}$ \\
\hline
\end{tabular}

altagahizi, nouvelle espèce apparentée au groupe orcinus s.l. Description de cette espèce et comparaison avec l'autre espèce libanaise, $N$. nadarini.

\section{SUMMARY}

Description of a small warm cave situated along the Mediterranean shore; the 
temperature of water varies from $14^{\circ}$ to $19^{\circ} \mathrm{C}$. Inhabiting this cave is Niphargus altagahizi, a new species related to orcinus s.l. group. Description of this species and comparison with the other Lebanese species, $N$. nadarini.

\section{BIBLIOGRAPHIE}

ALOUF, N., 1972 - Description d'un nouvel Amphipode gammaridé des eaux souterraines du Liban, Niphargus nadarini. Ann. Spéléo., 27, sous presse.

BIRSTEIN, J.A., 1961 - Biospeologica sovietica, XIV. Les Amphipodes souterrains de Crimée. Buil Soc. Nat. Moscou, Sect. biol., LXVI.

CHAPPUIS, P.A. et IELAMARE DEBOUTTEVILLE, C., 1954 - Les Isopodes psammiques de la Méditerranée, in: Recherches sur les Crustacés souterrains. Arch. Zool. Exp. Gén., Tome 91, Biospeologica 74, 103-138.

COIFFAIT, H., 1954 - Contribution à la connaissance de la faune cavernicole et endogée du Liban. Arch. Zool. Exp. Gén., T. 91, Biospeologica 75, 333-364.

GINET, R., 1960 - Ecologie, Ethologie et Biologie de "Niphargus". Ann. Spéléol., XV, 1-2, $1-254$.

KARAMAN, S., 1950 - Etudes sur les Amphipodes - Isopodes des Balkans. Acad. Serbe Sci. Mono., CLXIII, n. 2 (nouvelle série), 101-174.

KARAMAN, G., 1968 - II. Beitrag zur Kenntnis der Amphipoden von Crna Gora. Fragmenta balcanica, Mus. Mac. Sci. Nat. Skopje, Vi, n. 11/146, 109-119.

RUFFO, S., VIGNA-TAGLIANTI, A., 1968 - Alcuni Niphargus delle acque sotterranee dell'Italia centro-meridionale e considerazioni sulla sistematica del gruppo orcinus. Mem. Mus. civ. St. Nat. Verona, XVI, 1-29.

SCHELLENBERG, A., 1937 - Niphargen (Amphipoden) des Französichen Jura und Jugoslawiens. Zool. Anz., 120, 7/8, 161-169.

SKET, B., 1959 - Einige neue Formen der Malacostraca aus Yugoslawien; II. Bull. Sci. Sect. A., T. $4,4$.

VANDEL, A., 1964 - Biospéologie, la biologie des animaux cavernicoles. Paris. Gauthier-Villars, éd.

Actes du premier Colloque International sur le genre Niphargus (1969). Mus. Civ. St. Nat. Verona, Série no. 5, 1972. 